\title{
On the Uniqueness of Traveling Forced Curvature Fronts in a Fibered Medium
}

\author{
Gawtum Namah*
}

ENSMM, 26 chemin de l'Epitaphe, 25000 Besançon, and LMB, UMR CNRS 6623,

Université de Bourgogne Franche-Comté, France.

Received December 21, 2017; Accepted April 27, 2018

\begin{abstract}
We investigate traveling fronts, including pulsating ones, of a forced curvature flow in a plane fibered medium. The main topic of this note is an uniqueness issue of such traveling fronts. In addition to line-shaped profiles, we also consider traveling fronts in the form of V-shaped parabolas.
\end{abstract}

AMS subject classifications: $35 \mathrm{~K} 55,35 \mathrm{~B} 10$

Key words: Traveling wave solutions, pulsating fronts, periodic fibered medium.

\section{Introduction}

In this note, we will be interested in traveling fronts of a forced curvature flow equation

$$
V_{n}=R+K
$$

in the plane containing periodic striations. $V_{n}$ is the normal velocity of a propagating interface $\Gamma(t), K$ is its mean curvature and $R$ is the driving force. For example if $\Gamma$ is a flame front, then $R$ corresponds to the combustion rate of the burning material. In all cases, we will suppose that the function $R$ is smooth and verifies

$$
0<R_{m} \leq R \leq R_{M}
$$

Before going further, let us give a definition of a traveling front of Eq. (1.1).

Definition 1.1. $\Gamma(t)$, solution of (1.1) will be called a traveling front if there exists a constant vector $\mathbf{v} \in \mathbb{R}^{2}$ such that

$$
\Gamma(t)=\Gamma_{0}+\mathbf{v} t
$$

for all $t \in \mathbb{R}$. Then $\Gamma_{0}$ is the (constant) profile of the traveling front and $|\mathbf{v}|$, its speed, see Figure 1.

*Corresponding author. Email address: gnamah@ens2m.fr (G. Namah) 
Note that if $\Gamma(t)$ can be represented by the graph of a function $u$ in the $x-y$ plane, for example

$$
\Gamma(t)=\{(x, y) / y=u(x, t)\},
$$

then $V_{n}$ is given by

$$
V_{n}=\frac{u_{t}}{\sqrt{1+u_{x}^{2}}}
$$

so that Equation (1.1) becomes

$$
u_{t}-R \sqrt{1+u_{x}^{2}}=\frac{u_{x x}}{1+u_{x}^{2}}, \quad t \in \mathbb{R}, x \in \mathbb{R} .
$$

Now if $\Gamma(t)$ is a traveling front in the plane, we can suppose without loss of generality that $\mathbf{v}$ is parallel to the $\mathrm{y}$-axis i.e. $\mathbf{v}={ }^{t}(0, c)$. Then $u(x, t)$ will be given by

$$
u(x, t)=c t+\varphi(x)
$$

so that Equation (1.3) becomes

$$
c-R \sqrt{1+\varphi_{x}^{2}}=\frac{\varphi_{x x}}{1+\varphi_{x}^{2}}, \quad x \in \mathbb{R} .
$$

In the above, $c$ is the speed and $\varphi$ the constant profile of the wave. The pair $(c, \varphi)$ will be called a traveling wave solution (TWS) of Eq. (1.3). Note that every solution $\varphi$ of (1.4) is defined up to an additive constant.

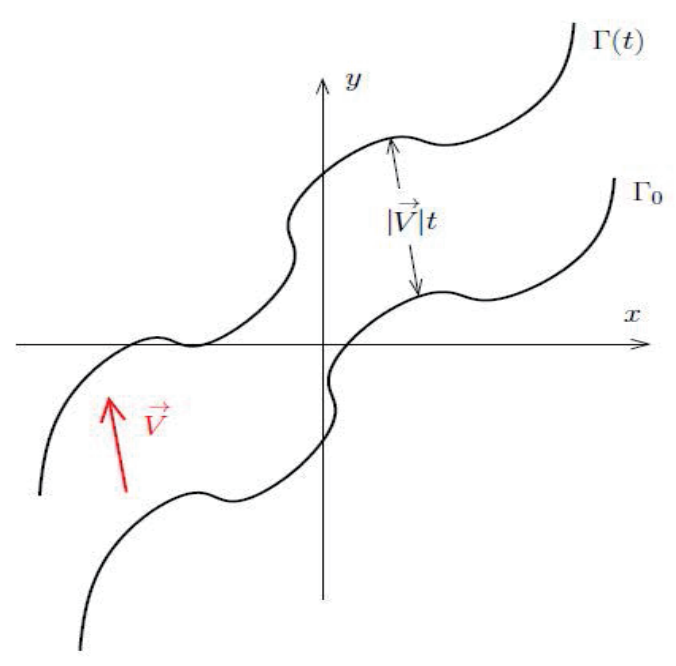

Figure 1: A TWS: a constant profile moving with a constant speed in some given direction. 
In [9], the authors gave a complete classification of smooth traveling fronts in the plane in the case of a constant $R$ i.e. for propagations in homogeneous media. They notably showed that a non stationary traveling front in the plane can only be a graph in the form of a line or of a V-shaped parabola. Different results have also been proved in the case of an interface propagating in a fibered medium with periodic striations (i.e. $R$ is periodic in one direction). In this case, one of course expects the lines and V-shaped fronts to become undulated, the results depending also on the angle of inclination of the striations. Let us precise what we mean by an undulated line-shaped front.

Definition 1.2. An undulated front will be termed line-shaped if there exists an angle $\alpha \in(-\pi / 2, \pi / 2)$ such that the front is at a finite distance from the line $y=\tan \alpha x$ for all $x \in \mathbb{R}$.

The line-shaped fronts can therefore be inclined (when $\alpha \neq 0$ ) or horizontal $(\alpha=0)$.

Essentially two types of solutions have been put forward in the case of a periodic fibered medium : traveling wave solutions (TWS) and pulsating traveling wave solutions (PTWS). Besides having some periodicity in space, these pulsating fronts also display a time periodicity namely there exists a period $T$ and some constant $Y$ such that

$$
u(x, t+T)=u(x, t)+Y, \quad \text { for all } t \in \mathbb{R} \text { and } x \in \mathbb{R} .
$$

Let us point out that equations of type (1.1) may exhibit PTWS in other contexts where the periodicity is not necessarily carried by the function $R$. See for example [7] and the references therein where it is the boundary of the corresponding domain which has periodic or quasi-periodic undulations while $R$ remains constant.

In [2], which deals with vertical striations $(R(x, y)=R(x), 1$-periodic $)$, it is shown that there exists a unique 'horizontal' line-shaped traveling front $(c, \varphi), \varphi$ being 1-periodic and $c$ the speed of the wave in the $y$ direction. The latter traveling front corresponds therefore to the undulated version of the horizontal line in the case of a constant $R$.

In the more recent work [6], the above result has been generalized to inclined fronts. The existence of inclined traveling fronts in the case of vertical striations is studied in an almost periodic context i.e. with $R$ almost periodic in $x$. Among other results, it is shown that for any $\alpha \in(-\pi / 2, \pi / 2), \alpha$ being the angle between the front line and the $x$ axis, there exists a unique $\left(c^{\alpha}, \varphi^{\alpha}\right), \varphi_{x}^{\alpha}$ almost periodic in $x$, solution of (1.4). The previous result easily leads to the existence of a unique traveling front $\Gamma^{\alpha}$ given by the pair $\left(c^{\alpha}, \varphi^{\alpha}\right)$, with $\varphi_{x}^{\alpha}$ periodic in the case of a periodic function $R$. Therefore we have a traveling front with $\Gamma_{0}=\left\{y=\varphi^{\alpha}(x)\right\}$ and $\mathbf{v}={ }^{t}\left(0, c^{\alpha}\right)$.

In the case of oblique striations treated in [8] and [3], the authors put in evidence the fact that periodicity in space generates a time periodic regime. More precisely, let us consider periodically disposed oblique striations of period 1 and inclined by an angle $\beta \in(0, \pi / 2)$ from the $y$-axis. Set

$$
X^{\beta}=1 / \cos \beta \text { and } Y^{\beta}=1 / \sin \beta \text {, }
$$


so that $R$ is $X^{\beta}$-periodic in $x$ and $Y^{\beta}$-periodic in $y$. Then there exists a unique $T^{\beta}$ and a unique function $\varphi^{\beta}(x, t), T^{\beta}$-periodic in time and $X^{\beta}$-periodic in $x$ such that

$$
u^{\beta}(x, t)=c^{\beta} t+\varphi^{\beta}(x, t),
$$

is a solution of (1.3), with $c^{\beta}=Y^{\beta} / T^{\beta}$. We thus have (see Figure 2)

$$
u^{\beta}\left(x, t+T^{\beta}\right)=u^{\beta}(x, t)+Y^{\beta} .
$$

Note that the uniqueness holds up to addition of constants in space and translations in time. The pair $\left(c^{\beta}, \varphi^{\beta}\right)$ or the moving front $u^{\beta}$ will be called a pulsating traveling wave solution (PTWS) of (1.3). Here $c^{\beta}$ represents the mean speed (over one time period) of the wave propagation in the $y$ direction.

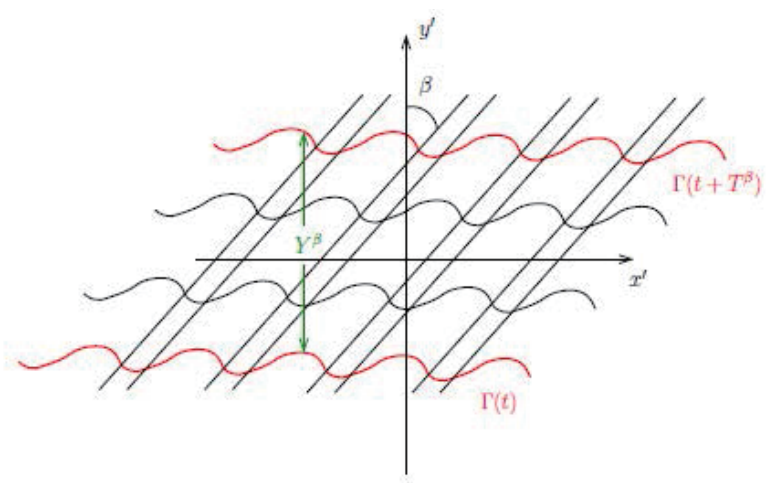

Figure 2: A horizontal line-shaped pulsating front : there exists $T^{\beta}$ such that for all $t, \Gamma\left(t+T^{\beta}\right)=\Gamma(t)+Y^{\beta}$.

Note also that, as $u^{\beta}$ does not have a constant profile, it is not clear here whether this pulsating front is a traveling wave solution. That this is the case will be the object of the following result :

Claim 1. Let $\Gamma(t)=\{y=u(x, t)\}$ be a space-periodic solution of (1.1) in a periodic fibered medium such that

$$
u(x, t+T)=u(x, t)+Y, \quad x \in \mathbb{R}, t \in \mathbb{R},
$$

for some given $T>0$ and $Y \in \mathbb{R}$. Then $\Gamma(t)$ slides with a constant speed along the direction of the striations.

The above result implies that any periodic pulsating front is a traveling wave solution i.e. propagating with a constant speed in a given direction, here that of the striations.

Now we want to enquire about the 'uniqueness' of these TWS. More precisely, consider a vertically striated medium in the $x-y$ plane. Recall that for any given $\alpha \in(-\pi / 2, \pi / 2)$, there exists a line-shaped traveling front $\Gamma^{\alpha}$ in the form of a graph $\left\{y=\varphi^{\alpha}(x)\right\}$ traveling with speed $c^{\alpha}$ in the $y$-direction. Can there exist a line-shaped traveling wave $\Gamma^{\prime}(t)=\left(\Gamma_{0}^{\prime}, \mathbf{v}^{\prime}\right)$ other than the $\left(\Gamma^{\alpha}\right)_{\alpha}$ ? For example $\Gamma^{\prime}$ may be a graph in some frame $x^{\prime}-$ $y^{\prime}$ other than $x-y$ and /or $\mathbf{v}^{\prime}$ may not be parallel to the $y$ axis.

Our aim in this paper is to clarify this uniqueness issue. We will notably show 
Theorem 1.1. Let $R=R(x), 1$-periodic in the $x$-y frame. Let $\Gamma^{\prime}(t)$ be a traveling wave solution of (1.1) in the form of a line-shaped graph in some $x^{\prime}-y^{\prime}$ frame. Then there exists $\alpha \in(-\pi / 2, \pi / 2)$, such that $\Gamma^{\prime}=\Gamma^{\alpha}$.

Therefore the $\left(\Gamma^{\alpha}\right)_{\alpha}$ are the only line-shaped traveling fronts which can exist. But as pointed out before, there exist also traveling fronts in the form of V-shaped graphs. Let us recall that in the homogeneous case ( $R=$ constant), (c.f. [9]), for any $\alpha \in(0, \pi / 2)$ there exists a unique function $\psi^{\alpha}$ in the form of a V-shaped parabola which has as asymptotes $\psi_{+}(x)=\tan \alpha x$ and $\psi_{-}(x)=-\tan \alpha x$ when $x \longrightarrow \pm \infty$, such that

$$
\Gamma_{0}=\left\{y=\psi^{\alpha}(x)\right\} \text { and } c^{\alpha}=R / \cos \alpha .
$$

Note that such a $\Gamma_{0}$ is symmetrical wrt the $y$ axis and $\psi_{x}^{\alpha}(0)=0$. The result corresponding to a striated medium goes as follows:

Theorem 1.2. Let $R=R(x)$ be 1-periodic. Let $\alpha \in(0, \pi / 2)$ satisfy $R_{M} \cos \alpha<R_{m}$. Then for any $s \in \mathbb{R},(1.4)$ admits a unique $V$-shaped traveling wave solution $\psi^{\alpha}$ such that $\psi^{\alpha}(0)=s$ and $\psi_{x}^{\alpha}(0)=0$.

In Section 2, we will deal with line-shaped traveling fronts. We will notably show that periodic pulsating fronts (other than straight lines) in a periodic fibered medium are just traveling fronts which slide down along the direction of the striations (Claim 1) and then prove Theorem 1.1. In Section 3, we will prove Theorem 1.2 concerning V-shaped traveling fronts.

\section{Line-shaped traveling fronts}

\subsection{The case of a homogeneous medium}

Let us start by noting that when $R$ is a constant, there is an infinite number of lines which are traveling fronts. Indeed for any $\alpha \in(-\pi / 2, \pi / 2)$, the moving line $\Gamma(t)=\Gamma_{0}+\mathbf{v} t$ with

$$
\left.\Gamma_{0}=\{y=\tan \alpha x)\right\} \text { and } \mathbf{v}={ }^{t}(-R \sin \alpha, R \cos \alpha),
$$

is a solution. Moreover the velocity $\mathbf{v}$ is not unique. In the above, we see that the line propagates perpendicular to itself but we could also have considered propagations in other directions. For example if we consider the line to be propagating in the $y$-direction, then $\mathbf{v}={ }^{t}(0, R / \cos \alpha)$. Likewise $\mathbf{v}={ }^{t}(-R / \sin \alpha, 0)$ for a propagation in the $x$-direction. Also, in this simple case, $\Gamma_{0}$ can also be written as a function of $y$ i.e. $\left.\Gamma_{0}=\{x=\cot \alpha y)\right\}$ for any $\alpha \neq 0$.

\subsection{The case of a periodic striated medium}

Let us now suppose that $R$ is 1-periodic in some direction in the plane, which can be taken to be the $x$ direction without any loss of generality, and is constant along the $y$ direction. We look for line-shaped traveling wave solutions of (1.4). We state the following 
Proposition 2.1. Let $R=R(x)$, 1-periodic. For any $\alpha \in(-\pi / 2, \pi / 2)$, there exists a unique line-shaped TWS $\Gamma^{\alpha}$ in the form of a graph $y=u(x, t)$, with $u$ satisfying Equation (1.3) and the shifted periodic condition

$$
u(x+1, t)=u(x, t)+\tan \alpha \text { for all real } t .
$$

In other words, there exists a unique pair $(c, \varphi)$ solution of

$$
c-R \sqrt{1+\varphi_{x}^{2}}=\frac{\varphi_{x x}}{1+\varphi_{x}^{2}}, \quad x \in \mathbb{R},
$$

such that

$$
\varphi(x+1)=\varphi(x)+\tan \alpha .
$$

Moreover we have the following estimates on $c$ and $\varphi$ :

$$
\begin{aligned}
& \frac{R_{m}}{\cos \alpha} \leq c \leq \frac{R_{M}}{\cos \alpha}, \\
& \left|\varphi_{x}(x)\right|<\sqrt{\frac{R_{M}^{2}}{R_{m}^{2} \cos ^{2} \alpha}-1 .}
\end{aligned}
$$

Of course, $c$ and $\varphi$ depend on $\alpha$. We have omitted the superscript $\alpha$ as there is no ambiguity. As usual, the uniqueness of $\varphi$ is to be understood up to an additive constant. The traveling front is therefore either periodic (when $\alpha=0$ ) or shifted-periodic in which case $\varphi$ is not periodic but it is $\varphi_{x}$ which is a 1-periodic function with $\int_{0}^{1} \varphi_{x} d x=\tan \alpha$.

Proof. Consider the ode satisfied by $h=\varphi_{x}$ and set

$$
f(x, h, c)=c\left(1+h^{2}\right)-R\left(1+h^{2}\right)^{3 / 2} .
$$

Then showing the above proposition comes to proving that

$$
\left\{\begin{array}{l}
h^{\prime}=f(x, h, c), \quad x \in(0,1) \\
h(0)=h(1) \\
\int_{0}^{1} h d x=\tan \alpha
\end{array}\right.
$$

admits a unique solution $(c, h)$. The proof will be omitted as it goes almost along the same lines as in [2] which was run for $\alpha=0$.

We can further estimate $h$ and give a monotonicity result on $c$ under some restrictions on $\alpha$. We state

Proposition 2.2. Let $(c, h)$ be the solution of (2.2). Then if $R_{M} \cos \alpha<R_{m}$, we have

$$
\begin{aligned}
& h(x) \geq \sqrt{\frac{R_{m}^{2}}{R_{M}^{2} \cos ^{2} \alpha}-1}, \quad \text { for all } x \in[0,1], \\
& c^{\prime}(\alpha)>0 .
\end{aligned}
$$


Proof. Here too, we will omit the proof as it is similar to the one given in [6] in the case of almost periodic striations.

Remark 2.1. Let us remark that the result of Proposition 2.1 excludes the case of $\alpha= \pm \pi / 2$. In fact, we know that for $\alpha=\pi / 2$ (see, e.g., [1]), there exists no TWS but a unique pulsating front which is a straight line propagating with an effective speed given by the harmonic mean $\overline{\bar{R}}$ of $R$. We thus have a front of the form $x=U(t)$ with $U(t+T)=U(t)+1$ with $T$ given by $T=1 / \overline{\bar{R}}$.

\subsection{Proof of Claim 1}

Consider a propagation through a 1-periodic fibered medium with the striations inclined by some angle $\beta \in(-\pi / 2, \pi / 2)$ from the $y$ axis, see Figure 2 . Here Eq. (1.3) reads

$$
u_{t}-R(x, y=u(x, t)) \sqrt{1+u_{x}^{2}}=\frac{u_{x x}}{1+u_{x}^{2}}, \quad t \in \mathbb{R}, x \in \mathbb{R}
$$

with

$$
R(x, y)=R(y \sin \beta-x \cos \beta) .
$$

Then we know (see, e.g., $[3,8]$ ) that there exists a unique $c^{\beta}>0$ and a unique space-time periodic function $\varphi^{\beta}$ such that

$$
u(x, t)=c^{\beta} t+\varphi^{\beta}(x, t)
$$

is a solution of (2.3). The front $u$ is therefore a periodic pulsating one i.e. there exists $T^{\beta}=$ $Y^{\beta} / c^{\beta}$ such that

$$
u\left(x, t+T^{\beta}\right)=u(x, t)+Y^{\beta} .
$$

We proceed by the following

Lemma 2.1. Let $u$ be a periodic pulsating front solution of (2.3)-(2.4). Then there exists a $X^{\beta}$ periodic function $W$ and a positive constant $c$ such that

$$
u(x, t)=W(x-c t \sin \beta)+c t \cos \beta .
$$

Note that Lemma 2.1 implies that the front slides along the direction of the striations with the constant speed $c$ without changing its profile as in Figure 2. We are therefore in the presence of a traveling front with

$$
\Gamma_{0}=\{y=W(x)\} \text { and } \mathbf{v}={ }^{t}(c \sin \beta, c \cos \beta),
$$

in the $x-y$ plane. 
Proof. So let $u=u(x, t)$ be a PTWS of (2.3)-(2.4). Note that in the present case, $R$ is also given by

$$
R(x, y)=R(x+s \sin \beta, y+s \cos \beta), \text { for all } s \in \mathbb{R} .
$$

Consider now, for $s \in \mathbb{R}$, the function

$$
W^{s}(x, t)=u(x-s \sin \beta, t)+s \cos \beta .
$$

It is then not difficult to verify that $W^{s}$ satisfies Equation (2.3). Indeed, by taking the Equation (2.3) at the point $(x=x-s \sin \beta, t=t)$, we get

$$
W_{t}^{s}(x, t)-R(x-s \sin \beta, y=u(x-s \sin \beta, t)) \sqrt{1+W_{x}^{s 2}}=\frac{W_{x x}^{s}}{1+W_{x}^{s 2}}, \quad t \in \mathbb{R}, x \in \mathbb{R} .
$$

But as $R$ is constant in the direction of the striations, we have

$$
\begin{aligned}
& R(x-s \sin \beta, y=u(x-s \sin \beta, t)) \\
= & R(x, u(x-s \sin \beta, t)+s \cos \beta)=R\left(x, W^{s}(x, t)\right),
\end{aligned}
$$

so that finally $W^{s}$ satisfies

$$
W_{t}^{s}(x, t)-R\left(x, W^{s}(x, t)\right) \sqrt{1+W_{x}^{s 2}}=\frac{W_{x x}^{s}}{1+W_{x}^{s 2}}, \quad t \in \mathbb{R}, x \in \mathbb{R} .
$$

Moreover we have

$$
W^{s}\left(x, t+T^{\beta}\right)=W^{s}(x, t)+Y^{\beta} .
$$

Therefore $W^{s}$ is PTWS of (2.3). By the uniqueness result of [8], we deduce that for any real $s, W^{s}$ is just a shift in time of $u$, i.e. there exists (a constant) $\tau(s)$ such that

$$
W^{s}(x, t)=u(x, t+\tau(s)) .
$$

We conclude from (2.7) that for all $s \in \mathbb{R}$, we have the following oblique shift for $u$ :

$$
u(x, t+\tau(s))=u(x-s \sin \beta, t)+s \cos \beta .
$$

Now due to the continuity of $\tau$ with respect to $s$ and the fact that $\tau(s) \longrightarrow \pm \infty$ when $s$ goes to $\pm \infty$, we can deduce that for any real $\tau$, there exists a real $s$ such that the above equation holds. Then for all real $\tau$, the front at time $t+\tau$ is just an oblique shift of the front at time $t$ along the direction of the striations.

Let $W=W(z)$ be the profile of the pulsating front at some reference time set to zero for simplicity. Then the front at any time $t>0$ is obtained by a shift of $W$ along the direction of the striations by a quantity $s=s(t)$, i.e.

$$
u(x, t)=W(x-s(t) \sin \beta)+s(t) \cos \beta .
$$


As $u$ satisfies (2.3), $W$ is therefore a solution of

$$
s^{\prime}(t)\left\{\cos \beta-\sin \beta W_{z}\right\}-R(z+s(t) \sin \beta, W(z)+s(t) \cos \beta) \sqrt{1+W_{z}^{2}}=\frac{W_{z z}}{1+W_{z}^{2}},
$$

which from (2.6) gives

$$
s^{\prime}(t)\left\{\cos \beta-\sin \beta W_{z}\right\}-R(z, W(z)) \sqrt{1+W_{z}^{2}}=\frac{W_{z z}}{1+W_{z}^{2}}, \quad t>0, z \in \mathbb{R} .
$$

But as $W$ is independent of time, we deduce that $s^{\prime}(t)$ is a constant so that there exists some real $c$ with $s(t)=c t$. We finally end up with

$$
u(x, t)=W(x-c t \sin \beta)+c t \cos \beta,
$$

and the proof of the Lemma is done and so is that of Claim 1. Note that in terms of $c^{\beta}$ (which we recall was the mean speed in the $y$ direction), we have $c^{\beta}=c \cos \beta$ so that $\mathbf{v}={ }^{t}\left(c^{\beta} \tan \beta, c^{\beta}\right)$.

\subsection{Proof of Theorem 1.1}

Let $\Gamma^{\prime}(t)$ be a TWS of (1.3) in the form of a line-shaped graph in some tilted $x^{\prime}-y^{\prime}$ frame and let $\beta \in(-\pi / 2, \pi / 2), \beta \neq 0$, be the angle between the $x^{\prime}$ and the $x$ axes, i.e. between the two frames. We will deal with the case $\beta= \pm \pi / 2$ afterwards. Recall that the striations are vertical in the original $x-y$ frame so that they will be oblique inclined by an angle $\beta$ from the $y^{\prime}$-axis.

Set $\Gamma^{\prime}(t)=\left\{y^{\prime}=u\left(x^{\prime}, t\right)\right\}$. The front $u$ is therefore a solution of (2.3)-(2.4). Now we can have a 'horizontal' front in the $x^{\prime}-y^{\prime}$ frame in which case $u$ will be periodic in $x^{\prime}$, or an 'inclined front'. We will first show that the traveling wave front $\Gamma^{\prime}(t)$ is in fact a PTWS which propagates along the direction of the striations and then prove that it coincides with one of the fronts $\Gamma^{\alpha}$ of Proposition 2.1. Let us start by considering the case of a horizontal front.

\subsubsection{Case $1: \Gamma^{\prime}$ horizontal in the tilted frame}

Note that as $\Gamma^{\prime}$ is a TWS, it propagates with a constant speed $|\mathbf{v}|$ but we do not know at this stage whether the direction of $\mathbf{v}$ is the same as that of the striations. But since $u$ is periodic in $x^{\prime}$, we can say that there exists some $T>0$ (in fact here $T$ will be given by $X^{\beta} / \mathbf{v}_{x^{\prime}}$ where $\mathbf{v}_{x^{\prime}}$ is the component of the velocity $\mathbf{v}$ in the $x^{\prime}$ direction) such that

$$
u\left(x^{\prime}, t+T\right)=u\left(x^{\prime}, t\right)+Y
$$

with $Y=\mathbf{v}_{y^{\prime}} T$. There is therefore a vertical shift (in the direction of $y^{\prime}$ ) of the front after time T. But as $u$ satisfies Eq. (2.3), we conclude that $\Gamma^{\prime}(t)$ is a PTWS of (2.3). Then we deduce by Claim 1 that $\mathbf{v}$ is parallel to the direction of the striations. Moreover by the uniqueness of the PTWS, we know that $\mathbf{v}={ }^{t}\left(c^{\beta} \tan \beta, c^{\beta}\right)$ with $c^{\beta}$ as previously defined. 
Now in the context of the original $x-y$ frame, $\Gamma^{\prime}$ is a traveling line-shaped front inclined by an angle $\beta$ from the $x$ axis and propagating with a constant speed in the $y$ direction. Now it suffices to prove that $\Gamma^{\prime}$ is a graph in the $x-y$ frame and conclude by the uniqueness result of Proposition 2.1 that $\Gamma^{\prime}$ coincides with $\Gamma^{\beta}$.

Take $t=0$ as a reference time and set $\Gamma_{0}^{\prime}=\Gamma^{\prime}(0)$. Define $u_{0}\left(x^{\prime}\right)=u\left(x^{\prime}, 0\right)$ so that $\Gamma_{0}^{\prime}=$ $\left\{y^{\prime}=u_{0}\left(x^{\prime}\right)\right\}$. We will prove that $\Gamma_{0}^{\prime}$ is a graph in the $x$-y coordinate frame. Indeed any point $\left(x^{\prime}, y^{\prime}\right)$ in the $x^{\prime}-y^{\prime}$ frame has as coordinates

$$
\begin{aligned}
& x=\cos \beta x^{\prime}-\sin \beta y^{\prime}, \\
& y=\sin \beta x^{\prime}+\cos \beta y^{\prime}
\end{aligned}
$$

in the $x-y$ coordinate frame so that any point $\left(x^{\prime}, u_{0}\left(x^{\prime}\right)\right)$ on $\Gamma_{0}^{\prime}$ reads

$$
\begin{aligned}
& x=\cos \beta x^{\prime}-\sin \beta u_{0}\left(x^{\prime}\right)=f\left(x^{\prime}\right), \\
& y=\sin \beta x^{\prime}+\cos \beta u_{0}\left(x^{\prime}\right)=g\left(x^{\prime}\right)
\end{aligned}
$$

in the $x-y$ frame. A sufficient condition to be able to write $y$ as a function of $x$ is that $f_{x}\left(x^{\prime}\right) \neq 0$ for all $x^{\prime}$. This ensures that we cannot have two values of $x^{\prime}$ corresponding to the same $x$. Otherwise, one can easily verify that this latter situation would lead to the case of having one value of $x$ corresponding to two different values of $y$.

We see from (2.8a) that showing that $f_{x}\left(x^{\prime}\right)$ does not vanish comes to proving that $u_{0 x^{\prime}}\left(x^{\prime}\right) \neq \cot \beta$ for all $x^{\prime}$. We therefore look for estimates on $u_{0 x^{\prime}}$. As we know that $\Gamma^{\prime}(t)$ slides in the direction of the striations with the speed $c=c^{\beta} \sec \beta$, we have for all $t>0(t=0$ being the reference time)

$$
u\left(x^{\prime}, t\right)=u_{0}\left(x^{\prime}-c t \sin \beta\right)+c t \cos \beta,
$$

that is

$$
u\left(x^{\prime}, t\right)=u_{0}\left(x^{\prime}-c^{\beta} t \tan \beta\right)+c^{\beta} t .
$$

We remark that the space-time periodic function $\varphi^{\beta}\left(x^{\prime}, t\right)$ given in $(2.5)$ is constant along the line $y^{\prime}=x^{\prime}-c^{\beta} t \tan \beta$ and is given by

$$
\varphi^{\beta}\left(x^{\prime}, t\right)=u_{0}\left(x^{\prime}-c^{\beta} t \tan \beta\right) .
$$

By setting $z=x^{\prime}-c^{\beta} t \tan \beta$, we see from (2.3) that $u_{0}$ satisfies

$$
c^{\beta}-c^{\beta} \tan \beta u_{0 z}-R\left(z, u_{0}(z)\right) \sqrt{1+u_{u_{0 z}}^{2}}=v \frac{u_{0 z z}}{1+u_{0 z}^{2}},
$$

subject to the periodic conditions

$$
u_{0}(0)=u_{0}\left(X^{\beta}\right) \text { and } u_{0 z}(0)=u_{0 z}\left(X^{\beta}\right) .
$$


Setting $h=u_{0 z}$, we obtain

$$
\left\{\begin{array}{l}
h^{\prime}=\left(1+h^{2}\right)\left(c^{\beta}-c^{\beta} \tan \beta h-R \sqrt{1+h^{2}}\right), z \in\left(0, X^{\beta}\right) \\
h(0)=h\left(X^{\beta}\right) \\
\int_{0}^{X^{\beta}} h d z=0 .
\end{array}\right.
$$

Set $h_{M}=\max _{z} h(z)=h\left(z_{M}\right)$, the existence of $z_{M}$ is ensured by the periodicity of $h$. By writing the equation at $z=z_{M}$, we have

$$
0=\left(1+h_{M}^{2}\right)\left(c^{\beta}\left(1-\tan \beta h_{M}\right)-R \sqrt{1+h_{M}^{2}}\right) .
$$

Suppose now that $h_{M} \geq \cot \beta$. As $c^{\beta}$ and $R$ are positive, the RHS will be negative which is not possible. Therefore we end up with $h(z)<\cot \beta$ for all $z \in \mathbb{R}$.

We have thus proved that $\Gamma^{\prime}(t)$ can be written as a line-shaped graph in the $x-y$ coordinate frame sliding in the direction of the striations with a constant speed. From the uniqueness result of Proposition 2.1, we conclude that $\Gamma^{\prime}(t)$ is a traveling front which coincides with one of the $\Gamma^{\alpha}(t)$ and here precisely with $\Gamma^{\beta}$.

\subsubsection{Case 2: $\Gamma^{\prime}$ inclined in the tilted frame}

Now let us consider the case where $\Gamma^{\prime}(t)=\left\{y^{\prime}=u\left(x^{\prime}, t\right)\right\}$ is an 'inclined' TWS in some $x^{\prime}-y^{\prime}$ coordinate frame traveling with velocity $\mathbf{v}^{\prime}=\left(\mathbf{v}_{x^{\prime}}^{\prime}, \mathbf{v}_{y^{\prime}}^{\prime}\right)$. Let $\beta$ be, as before, the angle between the tilted and the original frame and denote by $\gamma$, the angle of inclination of the front with respect to the $x^{\prime}$-axis, see Figure 3 .

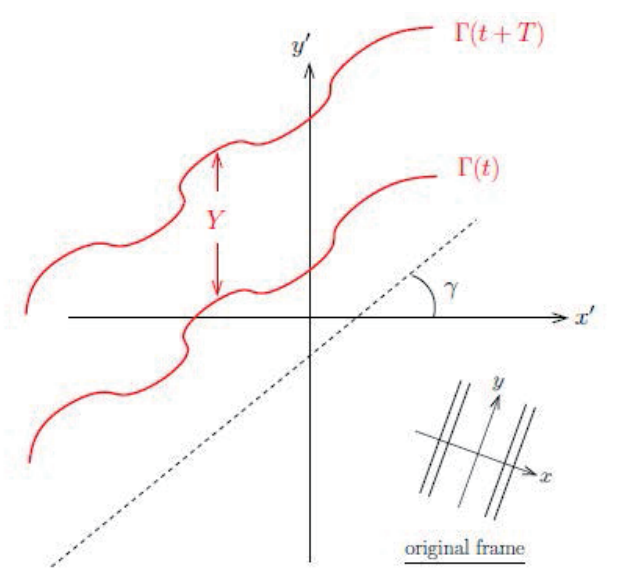

Figure 3: An inclined pulsating front in the tilted frame $x^{\prime}-y^{\prime}$ : there exists $T$ such that $\Gamma(t+T)=\Gamma(t)+Y$.

Recall that $u$ satisfies the same equation

$$
u_{t}-R\left(x^{\prime}, y^{\prime}=u\left(x^{\prime}, t\right)\right) \sqrt{1+u_{x^{\prime}}^{2}}=\frac{u_{x^{\prime} x^{\prime}}}{1+u_{x^{\prime}}^{2}}, \quad t \in \mathbb{R}, x^{\prime} \in \mathbb{R},
$$


as for the previous case but this time with shifted periodic conditions, namely

$$
u\left(x^{\prime}+X^{\gamma}, t\right)=u\left(x^{\prime}, t\right)+\tan \gamma X^{\gamma}
$$

for some $X^{\gamma}$. Here too, the periodicity in space (even if it is a shifted periodicity) will lead to a vertical shift of the front's profile after some time $T^{\prime}$, i.e. $\Gamma^{\prime}\left(t+T^{\prime}\right)$ is a vertical shift of $\Gamma^{\prime}(t)$ for all $t$, so that

$$
u\left(x^{\prime}, t+T^{\prime}\right)=u\left(x^{\prime}, t\right)+Y^{\prime},
$$

for some $Y^{\prime}$ which depends a priori on $\gamma$ and on the velocity $\mathbf{v}^{\prime}$. Here $T^{\prime}$ will be given by $T^{\prime}=X^{\gamma} / \mathbf{v}_{x^{\prime}}^{\prime}$. Let us start by remarking that, due to the periodicity of $R$, if $T^{\prime}$ exists then $Y^{\prime}$ is necessarily a multiple of $Y^{\beta}$. Indeed, consider the Equation (2.9) at the point $\left(x^{\prime}, t+T^{\prime}\right)$. We have

$$
u_{t}\left(x^{\prime}, t+T^{\prime}\right)-R\left(u\left(x^{\prime}, t+T^{\prime}\right) \sin \beta-x \cos \beta\right) \sqrt{1+u_{x^{\prime}}^{2}}\left(x^{\prime}, t+T^{\prime}\right)=\frac{u_{x^{\prime} x^{\prime}}}{1+u_{x^{\prime}}^{2}}\left(x^{\prime}, t+T^{\prime}\right) .
$$

But as $u_{t}\left(x^{\prime}, t+T^{\prime}\right)=u_{t}\left(x^{\prime}, t\right)$ and likewise for the derivatives in $x^{\prime}$, we have

$$
u_{t}\left(x^{\prime}, t\right)-R\left(u\left(x^{\prime}, t\right) \sin \beta-x \cos \beta+Y^{\prime} \sin \beta\right) \sqrt{1+u_{x^{\prime}}^{2}}\left(x^{\prime}, t\right)=\frac{u_{x^{\prime} x^{\prime}}}{1+u_{x^{\prime}}^{2}}\left(x^{\prime}, t\right),
$$

so that necessarily $Y^{\prime} \sin \beta \in \mathbb{Z}$. Thus $Y^{\prime}=m / \sin \beta=m Y^{\beta}$ for some $m \in \mathbb{Z}$.

The next step will be to verify that the inclined pulsating front slides along the direction of the striations with a constant speed (given by Claim 1 for the 'horizontal' case). Suppose this is true. Then we can run the proof almost along the same lines as that for the horizontal case to show that $\Gamma^{\prime}(t)$ is a graph in the original $x-y$ frame. Then from the uniqueness result of Proposition 2.1, we can again conclude that $\Gamma^{\prime}(t)$ coincides with one of the $\Gamma^{\alpha}(t)$, namely $\Gamma^{\beta+\gamma}(t)$ here. It now remains to verify that Claim 1 is also valid for an inclined front. What is important is the uniqueness result for pulsating fronts. In Claim 1 , the uniqueness result called for had been proved for periodic fronts (see, e.g., [4, 8]). Also in [8], the analysis was carried out for viscous Hamilton-Jacobi equations. In fact, the result holds in the present case too. For completeness sake, let us give a proof of the uniqueness of the pulsating front in the inclined case.

Suppose therefore that there exist two inclined pulsating fronts $u_{1}$ and $u_{2}$ solutions of (2.9)-(2.10). We know that we can write

$$
\begin{aligned}
& u_{1}\left(x^{\prime}, t\right)=c_{1} t+\varphi_{1}\left(x^{\prime}, t\right), \\
& u_{2}\left(x^{\prime}, t\right)=c_{2} t+\varphi_{2}\left(x^{\prime}, t\right),
\end{aligned}
$$

with $\varphi_{1}, T_{1}^{\prime}$-periodic (resp. $\varphi_{2}, T_{2}^{\prime}$-periodic) in time and both satisfying the shifted periodic condition (2.10). Let

$$
\begin{aligned}
& t_{1}=\sup \left\{t \in \mathbb{R} / u_{1}(., t)<u_{2}(., 0)\right\}, \\
& t_{2}=\inf \left\{t \in \mathbb{R} / u_{1}(., t)>u_{2}(., 0)\right\} .
\end{aligned}
$$


The quantity $t_{1}$ appears therefore as the first time that $u_{1}(., t)$ touches $u_{2}(., 0)$ from below and $t_{2}$ is the last time that $u_{1}(., t)$ touches $u_{2}(., 0)$ from above. We therefore have

$$
u_{1}\left(x^{\prime}, t_{1}\right) \leq u_{2}\left(x^{\prime}, 0\right) \text { and } u_{1}\left(x^{\prime}, t_{2}\right) \geq u_{2}\left(x^{\prime}, 0\right) \text {. }
$$

The weak maximum principles then lead to

$$
u_{1}\left(x^{\prime}, t_{1}+t\right) \leq u_{2}\left(x^{\prime}, t\right) \leq u_{1}\left(x^{\prime}, t_{2}+t\right) \quad \text { for all } t \in \mathbb{R},
$$

that is

$$
c_{1}\left(t_{1}+t\right)+\varphi_{1}\left(x^{\prime}, t_{1}+t\right) \leq c_{2} t+\varphi_{2}\left(x^{\prime}, t\right) \leq c_{1}\left(t_{2}+t\right)+\varphi_{1}\left(x^{\prime}, t_{2}+t\right) .
$$

Note that in the inclined case, the profiles $\varphi_{1}$ and $\varphi_{2}$ remain bounded in time from the line $y^{\prime}=\tan \gamma x^{\prime}$. Substract then $\tan \gamma x^{\prime}$ from each term, divide by $t$ and then let $t$ go to infinity. We obtain

$$
c_{1} \leq c_{2} \leq c_{1},
$$

so that $c_{1}=c_{2}$. The two fronts have therefore the same effective speeds. This in turn implies that the two time periods $T_{1}^{\prime}$ and $T_{2}^{\prime}$ are equal up to a multiplicative integer. More precisely, as we know that $Y_{2}^{\prime}=m Y_{1}^{\prime}$ for some $m \in \mathbb{N}$ (here we have supposed without loss of generality that $Y_{2}^{\prime} \geq Y_{1}^{\prime}$ ), then we will have $T_{2}^{\prime}=m T_{1}^{\prime}$.

Now let $x_{1}^{\prime}$ be such that $u_{1}\left(x_{1}^{\prime}, t_{1}\right)=u_{2}\left(x_{1}^{\prime}, 0\right)$. We have at the point $x^{\prime}=x_{1}^{\prime}$ and $t=t_{1,}$

$$
u_{1}\left(x_{1}^{\prime}, t_{1}+T_{2}^{\prime}\right)=c_{1} t+c_{1} T_{2}^{\prime}+\varphi_{1}\left(x_{1}^{\prime}, t_{1}+T_{2}^{\prime}\right)=c_{1} T_{2}^{\prime}+u_{1}\left(x_{1}^{\prime}, t_{1}\right) .
$$

Likewise at the point $x^{\prime}=x_{1}^{\prime}$ and $t=0$, we have

$$
u_{2}\left(x_{1}^{\prime}, T_{2}^{\prime}\right)=c_{2} T_{2}^{\prime}+u_{2}\left(x_{1}^{\prime}, 0\right),
$$

so that $u_{1}\left(x_{1}^{\prime}, t_{1}+T_{2}^{\prime}\right)=u_{2}\left(x_{1}^{\prime}, T_{2}^{\prime}\right)$. This situation is not allowed by the strong maximum principles applied to the linear parabolic equation satisfied by the function $w\left(x^{\prime}, t\right)$ given by

$$
w\left(x^{\prime}, t\right)=u_{1}\left(x^{\prime}, t+t_{1}\right)-u_{2}\left(x^{\prime}, t\right),
$$

unless $w$ is a constant.

\subsubsection{The case $\beta=\pi / 2$}

Note that Proposition 2.1 does not concern TWS in the form of line-shaped graphs $x=$ $W(y, t)$. In fact, this comes to considering a tilted frame $\left(x^{\prime}-y^{\prime}\right)$ with $y^{\prime}=x$ and $x^{\prime}=-y$, that is the angle $\beta$ between the two frames equals to $-\pi / 2$. So suppose there exists a TWS $\Gamma^{\prime}(t)$ in the form of a line-shaped graph $x=W(y, t)$ inclined by some angle $\alpha$ from the $x$-axis. We propose to show that $\Gamma^{\prime}$ coincides with the $\Gamma^{\alpha}$ given by the Proposition 2.1.

We know by Claim 1 that if it exists, $\Gamma^{\prime}(t)$ slides along the direction of the striations (here in the $y$-direction) with a speed $c$, which without loss of generality can be taken to be positive. Let us continue our analysis for $\alpha \in(0, \pi / 2)$, the case for negative values of 
$\alpha$ can be carried out in a similar way. We proceed by writing down the equation satisfied by $W$ :

$$
W_{t}+R(W) \sqrt{1+V_{y}^{2}}=\frac{V_{y y}}{1+V_{y}^{2}}, \quad t \in \mathbb{R}, y \in \mathbb{R} .
$$

Note the positive sign before $R$ in the above equation. Indeed as $\alpha>0$, a motion of the line $y=\tan \alpha x$ in the upward direction corresponds to a propagation in the negative $x$-direction. This gives a negative speed of the line in the $x$-direction. The sign would change for $\alpha<0$. Now as $\Gamma^{\prime}(t)$ has a constant profile moving with the speed $c$ there exists a function $\psi$ such that

$$
W(y, t)=\psi(y-c t),
$$

and where $\psi$ satisfies the following system :

$$
\begin{cases}-c \psi^{\prime}+R(\psi) \sqrt{1+\psi^{\prime 2}}=\frac{\psi^{\prime \prime}}{1+\psi^{\prime 2}}, & z \in \mathbb{R}, \\ \psi(z+\tan \alpha)=\psi(z)+1, & z \in \mathbb{R}, \\ \psi^{\prime}(z+\tan \alpha)=\psi^{\prime}(z), & z \in \mathbb{R} .\end{cases}
$$

Without loss of generality, as usual, we can normalize $\psi$ such that $\psi(0)=0$. We then state

Lemma 2.2. Let $\alpha \in(0, \pi / 2)$. If $\psi$ is a solution of $(2.12)$ then $\psi^{\prime}(z)>0$ for all $z \in \mathbb{R}$.

Proof. As $\psi^{\prime}$ is $\tan \alpha$-periodic, there exists $z^{*} \in(0, \tan \alpha]$ such that

$$
\psi^{\prime}\left(z^{*}\right)=\max _{\mathbb{R}} \psi^{\prime}(z)
$$

with $\psi^{\prime \prime}\left(z^{*}\right)=0$. As the first condition on $\psi$ implies that $\int_{0}^{\tan \alpha} \psi^{\prime}(z) d z=1(>0)$, necessarily we must have $\psi^{\prime}\left(z^{*}\right)>0$. By the equation of $\psi$, we recover the fact that $c>0$. Now let $z_{*}$ correspond to the minimum i.e. $\psi^{\prime}\left(z_{*}\right)=\min _{\mathbb{R}} \psi^{\prime}(z)$. Again by using the equation of $\psi$, we deduce that $\psi^{\prime}\left(z_{*}\right)>0$.

From the above lemma, we see that if a traveling front exists in the form of a graph $x=V(y, t)$, we will have $V(y, t)=\psi(y-c t)$ with $\psi$ invertible. Thus $\Gamma^{\prime}(t)$ may be written as $\Gamma^{\prime}(t)=\left\{y=c t+\psi^{-1}(x)\right\}$. We then conclude by the uniqueness result of Proposition 2.1 that $\Gamma^{\prime}=\Gamma^{\alpha}$ and we are done with the proof of Theorem 1.1.

\section{V-shaped TWS}

As mentioned before, there exist also traveling wave solutions in the form of V-shaped parabola. Let us first review the homogenous case before going to the uniqueness result in the fibered medium. 


\subsection{The homogeneous case}

Let $\alpha \in(0, \pi / 2)$ and consider the two lines $\psi_{+}(x)=\tan \alpha x$ and $\psi_{-}(x)=-\tan \alpha x$. Then there exists a unique V-shaped TWS

$$
\Gamma(t)=\Gamma_{0}+c t,
$$

with $c=R / \cos \alpha$ and $\Gamma_{0}=\{y=\psi(x)\}$ a V-shaped parabola which has $\psi_{+}(x)$ and $\psi_{-}(x)$ as asymptotes when $x \longrightarrow \pm \infty$ (cf. [9]). Note that $\Gamma_{0}$ is symmetrical with respect to the $y$ axis with $\psi^{\prime}(0)=0$.

\subsection{The periodic case}

Let us now consider the case where the forcing term $R(x, y)=R(x)$ is 1-periodic. We know from Proposition 2.1 that for every $\alpha \in(-\pi / 2, \pi / 2)$, there exists a unique lineshaped TWS $(c, \varphi)$ solving the problem

$$
P_{\alpha}:\left\{\begin{array}{l}
c-R \sqrt{1+\varphi_{x}^{2}}=\frac{\varphi_{x x}}{1+\varphi_{x}^{2}}, \quad x \in \mathbb{R} \\
\varphi(0)=0, \varphi(1)=\tan \alpha \\
\varphi^{\prime}(0)=\varphi^{\prime}(1) .
\end{array}\right.
$$

Remark 3.1. Note that the above boundary conditions are equivalent to the shifted periodic condition (2.1) of Proposition 2.1. Indeed if $(c, \varphi)$ solves $P_{\alpha}$, then we have

$$
\varphi(x+1)=\varphi(x)+\tan \alpha .
$$

To see this, consider $w(x)=\varphi(x+1)-\tan \alpha$. We then have $w(0)=0$ and $w^{\prime}(0)=\varphi^{\prime}(0)$. As $R$ is 1-periodic, $w$ also satisfies the first equation of $P_{\alpha}$. Then by uniqueness results for initial value problems for second order ode's, we know that $w \equiv \varphi$.

We now define for any function $\varphi$, the symmetrical function with respect to the $y$-axis $\varphi^{\text {sym }}$ i.e. $\varphi^{\text {sym }}(x)=\varphi(-x)$ and prove the following lemma:

Lemma 3.1. Let $\left(c_{1}, \varphi_{1}\right)$ and $\left(c_{2}, \varphi_{2}\right)$ be the respective line-shaped TWS of $P_{\alpha_{1}}$ and $P_{\alpha_{2}}$ with $\alpha_{1}>0$ and $\alpha_{2}<0$ and both satisfying the condition

$$
R_{M} \cos \alpha<R_{m}
$$

Then $\alpha_{1}=-\alpha_{2}$ iff $c_{1}=c_{2}$.

Proof. (i) $\alpha_{1}=-\alpha_{2} \Longrightarrow c_{1}=c_{2}$.

In the homogeneous case, it's trivial because $c=R / \cos \alpha$. When $R$ is periodic, without loss of generality, we can consider $R(x)=R(-x)$ for all $x$. Consider then $\psi=\varphi_{1}^{\text {sym }}$. It is not difficult to verify that $\psi$ satisfies

$$
\left\{\begin{array}{l}
c_{1}-R \sqrt{1+\psi_{x}^{2}}=\frac{\psi_{x x}}{1+\psi_{x}^{2}}, \quad x \in \mathbb{R}, \\
\psi(0)=0, \psi(1)=-\tan \alpha_{1}, \\
\psi^{\prime}(0)=\psi^{\prime}(1),
\end{array}\right.
$$


so $\left(c_{1}, \psi\right)$ is a solution of $P_{\alpha}$ for $\alpha=-\alpha_{1}$ i.e. for $\alpha=\alpha_{2}$. By uniqueness of line-shaped TWS, we deduce that $c_{1}=c_{2}$ and $\psi=\varphi_{2}=\varphi_{1}^{\text {sym }}$.

(ii) $c_{1}=c_{2} \Longrightarrow \alpha_{1}=-\alpha_{2}$.

Set $\beta=-\alpha_{2}$. We know that there exists a unique pair $\left(c_{\beta}, \varphi_{\beta}\right)$ solution of $P_{\beta}$. By (i), we then have $c_{\beta}=c_{2}$ and $\varphi_{\beta}=\varphi_{2}^{\text {sym }}$. As $c_{1}=c_{2}$ by assumption, then $c_{\beta}=c_{1}$. Now as $\alpha_{1}$ and $\beta$ satisfy assumption (3.1) and under the latter condition $c(\alpha)$ is strictly increasing in $\alpha$ (by Proposition 2.2), we have necessarily $\beta=\alpha_{1}$.

So for $\alpha \in(0, \pi / 2)$, the above lemma shows that the profile of the line-shaped TWS $\Gamma^{-\alpha}$ is symmetrical to that of $\Gamma^{\alpha}$, both having the same speed of propagation. It says a bit more notably that the only line-shaped TWS having the same speed as $\Gamma^{\alpha}$ is $\Gamma^{-\alpha}$. Now we are ready to give the result for V-shaped TWS.

Theorem 3.1. Let $\alpha \in(0, \pi / 2)$ satisfy assumption (3.1) and let $(c, \varphi)$ be the solution of $P_{\alpha}$ and $\varphi^{\text {sym }}$ be defined as above. Then for any $s \in \mathbb{R},(1.4)$ admits a unique $V$-shaped TWS $\psi$ such that $\psi(0)=s$ and $\psi^{\prime}(0)=0$. Moreover the solution satisfies the following estimates

(i) $\varphi^{\prime} \operatorname{sym}^{\prime}(x)<\psi^{\prime}(x)<\varphi^{\prime}(x)$,

(ii) there exist a unique $s_{0}>0$ and positive constants $L$ and $\delta$ such that $\psi(0)=s_{0}$ and

$$
\varphi(|x|)<\psi(x)<\varphi(|x|)+\operatorname{Lexp}(-\delta|x|) .
$$

Note that $\psi$ has $\varphi$ and $\varphi^{\text {sym }}$ as asymptotes when $x$ goes to $\pm \infty$. Note also that under assumptions (3.1) we can show that $\varphi^{\prime \text { sym }}(x)<0$ and $\varphi^{\prime}(x)>0$. We will omit the proof of the theorem as it goes along the same lines as Theorem $B$ of [6] by remarking that in the periodic case, $\alpha_{2}$ is necessarily equal to $-\alpha_{1}$ by Lemma 3.1. It suffices then to do the neccessary modifications.

\section{Acknowledgement}

The author would like to thank Nathaël Alibaud for our regular collaboration on problems related to propagation of fronts and for helpful discussions on this paper.

\section{References}

[1] C. M. Brauner, P. C. Fife, G. Namah and C. Schmidt-Lainé, Propagation of a combustion front in a striated solid medium: a homogenization analysis, Q. Appl. Math., LI (3) (1993), 467-493.

[2] X. Chen and G. Namah, Wave propagation under curvature effects in a heterogeneous medium, Appl. Anal., 64(3-4) (1997), 219-233.

[3] X. Chen and G. Namah, Periodic travelling wave solutions of a parabolic equation: a monotonicity result, J. Math. Anal. Appl., 275(2) (2002), 804-820. 
[4] N. Dirr, G. Karali and N.K. Yip, Pulsating wave for mean curvature flow in inhomogeneous medium, Eur. J. Appl. Math., 19 (2008), 661-699.

[5] B. Lou, Periodic travelling wave solutions of a curvature flow equation in the plane, Tohoku Math. J., 59 (2007), 365-377.

[6] B. Lou and X. Chen, Traveling waves of a curvature flow in almost periodic media, J. Differ. Equations, 247(8) (2009), 2189-2208.

[7] H. Matano, K-I. Nakamura and B. Lou, Periodic traveling waves in a two-dimensional cylinder with saw-toothed boundary and their homogenization limit, Netw. Heterog. Media, 1(4) (2006), 537-568.

[8] G. Namah and J.-M. Roquejoffre, Convergence to periodic fronts in a class of semilinear parabolic equations, Nodea.-Nonlinear. Diff., 4(4) (1997), 521-536.

[9] H. Ninomiya and M. Taniguchi, Traveling curved fronts of a mean curvature flow with constant driving force, Free Boundary Problems : Theory and Applications I (Chiba, 1999), 206-221, Gakuto Internat. Ser. Math. Sci. Appl., 13 Gakkotosho, Tokyo, 2000.

[10] J. Xin, Front propagation in heterogeneous media, SIAM Rev., 42(2) (2000), 161-230. 